\title{
ANÁLISE DE DADOS DE PRECIPITAÇÃO ESTIMADOS PELO SATÉLITE TRMM PARA O VALE DO MÉDIO PARAPANEMA - SP ${ }^{1}$
}

Daniela Fernanda da Silva ${ }^{2}$; Angélica Prela-Pantano ${ }^{3}$; Marcelo Bento Paes de Camargo ${ }^{4}$

\section{RESUMO}

As estimativas de precipitação por satélites vêm contribuindo para aumentar a cobertura de dados climatológicos. Dessa forma a utilização dos dados de precipitação estimados pelo satélite Tropical Rainfall Measuring Mission (TRMM) podem ser utilizados na ausência de dados das estações meteorológicas convencionais de superfície. Objetivou-se com este trabalho avaliar as estimativas de precipitação através do satélite TRMM para a região do Vale do Médio Paranapanema em comparação aos dados observados em estações de superfície, em escala decendial, para o período de 1998 à 2008. Os resultados obtidos foram satisfatórios, mostrando que as estimativas de precipitação mediante a utilização do satélite TRMM podem ser uma fonte confiável de dados quando não se dispõe de informações de estações de superfície

Palavras-chave: agrometeorologia, chuva, dados espectrais, estações meteorológicas,

\section{ABSTRACT \\ ANALYSIS OF PRECIPTATION DATA ESTIMATED BY THE TRMM SATELLITE FOR THE MIDDLE PARANAPANEMA BASIN - SP}

Precipitation estimates by satellites contribute to increased coverage of climate data. Thus, the use of rainfall data estimated by the Tropical Rainfall Measuring Mission satellite (TRMM) may be used in the absence of data from conventional meteorological surface stations. The objective of this study was to evaluate the precipitation estimates by the TRMM satellite for the middle Paranapanema basin compared to data observed by surface stations, on a decendial scale, for the period of 1998 to 2008 . The results obtained were satisfactory, showing that precipitation estimates based on using the TRMM satellite can be a reliable data source when no information is available from surface stations.

Keywords: meteorological stations, rain, spectral data, agrometeorology.

\section{Recebido para publicação em 08/02/2012. Aprovado em 14/02/2013.}

1 - Pesquisa integrante da dissertação de Mestrado, financiado pela FAPESP, CNPq

2 - Geógrafa, M.Sc. em Agricultura Tropical e Subtropical, Doutoranda em Eng. Agrícola, Feagri - UNICAMP, SP, silva.danielaf@gmail.com

3 - Eng. Agra. Dra em Física do Ambiente Agrícola, Pesquisadora Cientifica, Inst. Agronômico de Campinas, aprela@iac.sp.gov.br

4 - Eng. Agro. Dr. em Agrometeorologia, Pesquisador Científico, Instituto Agronômico de Campinas, mcamargo@iac.sp.gov.br

\section{REVENG}

138-147p. Engenharia NA Agricultura, VIÇOSA - MG, V.21 N.2, MARÇO / ABRIL 2013 


\section{INTRODUÇÃO}

Nos últimos anos observou-se considerável crescimento na utilização de sensoriamento remoto para estimativa de precipitação pluvial, aumentando assim a quantidade de informações meteorológicas disponíveis para uma determinada região.

Alguns trabalhos apresentam boa acurácia das estimativas de precipitação com dados oriundos do satélite TRMM (Tropical Rainfall Measuring Mission), por meio do sensor PR, como os de: Short et al., (2000); Cage et al., (2002); Lyu et al., (2003); Nobrega (2008); Dubreuil et al., (2007); Garcia et al., (2009); Leivas et al., (2009) e Bardin et al., (2010).

O satélite TRMM, está em órbita desde novembro de 1997, em uma parceria entre a NASA (National Aeronautics and Space Administration) e a JAXA (Agência Japonesa de Exploração Aeroespacial), com objetivo específico de monitorar e estudar a precipitação nas áreas tropicais (COLLISCHONN et al., 2007).

Os instrumentos a bordo do satélite TRMM são: imageador de microondas (TMI), radar de precipitação (PR), radiômetro no visível e no infravermelho (VRS), sensor de energia radiante da superfície terrestre e das nuvens (CERES), e sensor imageamento de relâmpagos (LIS), sendo o radar de precipitação (PR), que é o primeiro radar meteorológico a ser lançado a bordo de um satélite, permitindo produzir mapas tridimensionais de estruturas precipitantes.

O satélite possui órbita oblíqua nãoheliossíncrona bastante baixa (inicialmente 350 $\mathrm{km}$, desde 2001 cerca de $403 \mathrm{~km}$ ), de forma que o período de translação é bastante curto (91 minutos), permitindo resoluções espacial e temporal comparativamente altos. A órbita deste satélite foi calculada para se ter uma capacidade máxima de amostragem diurna e os dados são estimados a cada 3 horas com uma resolução espacial de $0,25^{\circ}$ desde $50^{\circ} \mathrm{N}$ a $50^{\circ} \mathrm{S}$.

Barrera (2005), afirma que em função de ser o satélite melhor equipado em termos de instrumentos para estimativa de precipitação, o satélite TRMM fornece estimativas mais precisas do que as técnicas indiretas, baseadas em imagens de outros satélites. As estimativas de precipitação oriundas do TRMM são consistentes, mostrando boa acurácia com o regime pluviométrico registrados em superfície, podendo afirmar que dados de satélite são uma alternativa para obtenção de dados de superfície (LEIVAS et al., 2009).
As estimativas espaciais de precipitação constituem uma ferramenta extremamente útil, embora pouco precisas quando comparadas com dados pontuais medidas no solo, pois a precipitação é a variável que apresenta maior variabilidade espacial (CAMARGO et al., 2005), e as estações meteorológicas convencionais fornecem registros válidos apenas para o seu entorno. Collischonn et al. (2007), realizou estudos na bacia do Paraguai Superior, onde analisaram e compararam os dados de campo em relação aos dados obtidos pelo satélite TRMM, e consideraram os resultados obtidos satisfatórios, mostrando que as estimativas deste satélite podem ser uma fonte alternativa de dados em escassez de informação.

Devido a escassez de estações de superfície na região de estudo, o objetivo do trabalho foi avaliar as estimativas de precipitação do satélite TRMM para região do Vale do Médio Paranapanema, e verificar a confiabilidade em relação aos dados observados em estações meteorológicas de superfície existentes, para o período de 1998 a 2008 , em escala decendial.

\section{MATERIAL E MÉTODOS}

A região encontra-se a sudoeste do estado de São Paulo entre os quadrantes de coordenadas geográficas $22^{\circ} 00^{\prime} \mathrm{S}$ a $23^{\circ} 00^{\prime} \mathrm{S}$ de latitude e $51^{\circ} 00^{\prime}$ $\mathrm{W}$ a $50^{\circ} 00^{\prime} \mathrm{W}$ de longitude (Figura 1 ).

A temperatura média da região fica em torno de $20,6{ }^{\circ} \mathrm{C}$ e a precipitação pluvial anual $1.217 \mathrm{~mm}$ (PRADO et al., 2003). Segundo a classificação de Köppen predomina na maior parte da área o clima tipo "Cwa" (definido como mesotérmico de inverno seco), porém a região se encontra em uma área de transição climática para "Cfa" (mesotérmico e úmido).

Os dados meteorológicos referentes à precipitação pluvial foram obtidos pelo sensor PR do satélite TRMM, etambém pormeio de estações meteorológicas convencionais de superfície, monitoradas pelo CIIAGRO-IAC (Centro Integrado de Informações Agrometeorológicas - Instituto Agronômico de Campinas). Após a obtenção dos dados de precipitação, os mesmos foram agrupados em decêndios, para os dois métodos de aquisição de dados.

Para obtenção dos dados pelo TRMM, foram utilizadas as coordenadas geográficas de cada estação de superfície com os pontos de coordenadas mais próximos do TRMM, para o período compreendido entre janeiro de 1998 a dezembro de 2008, para sete municípios (Quadro 1). 


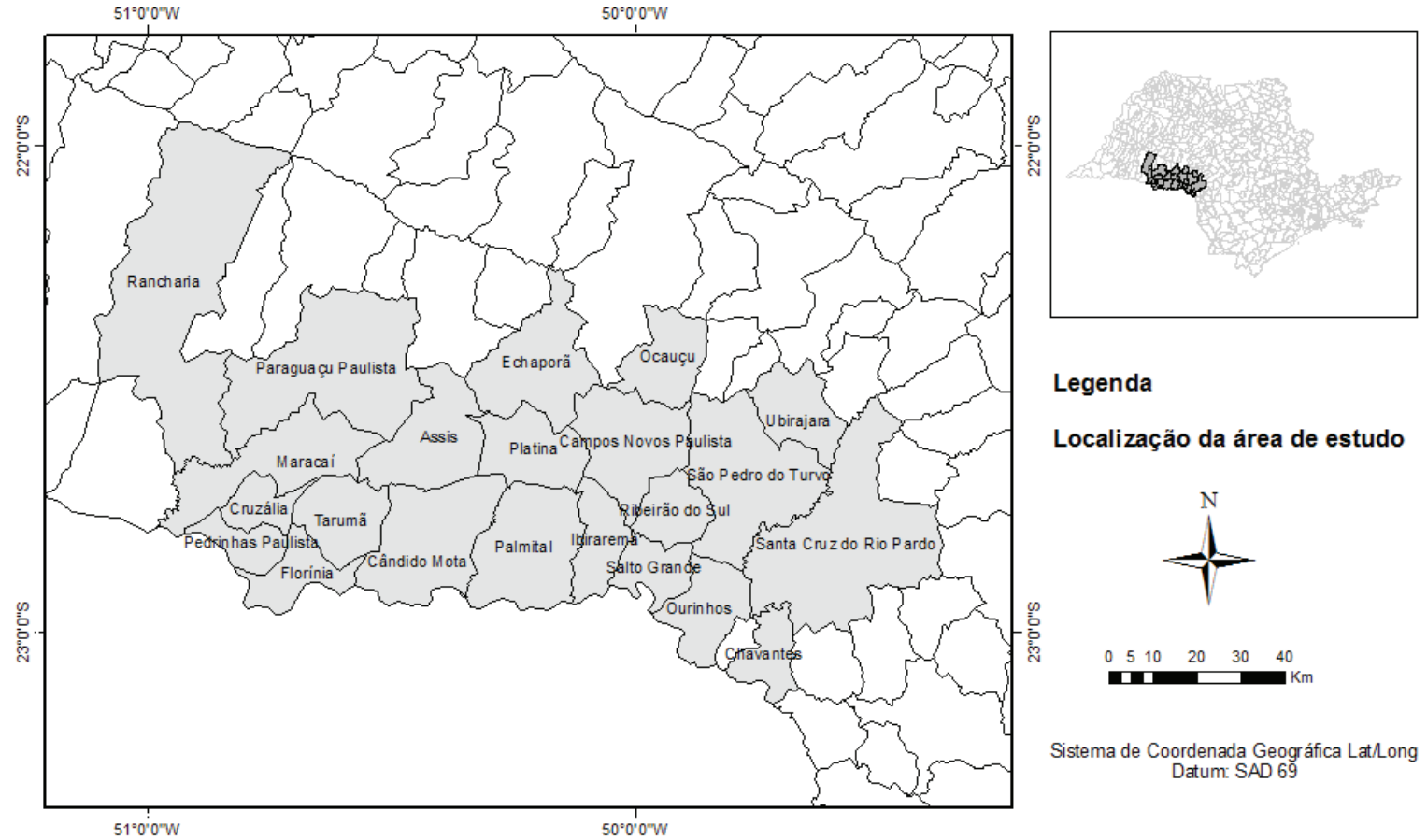

Figura 1. Localização da área de estudo.

Quadro 1. Localização geográfica dos postos meteorológicos de superfície do CIIAGRO/IAC, e do TRMM

\begin{tabular}{|c|c|c|c|c|c|}
\hline \multirow{2}{*}{ Municípios } & \multicolumn{2}{|c|}{ Estações de superfície } & \multirow[b]{2}{*}{$\begin{array}{l}\text { Altitude } \\
\text { (m) }\end{array}$} & \multicolumn{2}{|c|}{ Satélite TRMM } \\
\hline & $\begin{array}{c}\text { Latitude } \\
\text { (graus) }\end{array}$ & $\begin{array}{l}\text { Longitude } \\
\text { (graus) }\end{array}$ & & $\begin{array}{c}\text { Latitude } \\
\text { (graus) }\end{array}$ & $\begin{array}{l}\text { Longitude } \\
\text { (graus) }\end{array}$ \\
\hline Assis & $22,66 \mathrm{~S}$ & $50,43 \mathrm{~W}$ & 563 & $22,55 \mathrm{~S}$ & $50,45 \mathrm{~W}$ \\
\hline Campos Novos Paulista & $22,58 \mathrm{~S}$ & $50,00 \mathrm{~W}$ & 460 & $22,55 \mathrm{~S}$ & $50,20 \mathrm{~W}$ \\
\hline Cândido Mota & $22,76 \mathrm{~S}$ & $50,40 \mathrm{~W}$ & 472 & $22,75 \mathrm{~S}$ & $50,45 \mathrm{~W}$ \\
\hline Florínea & $22,91 \mathrm{~S}$ & $50,75 \mathrm{~W}$ & 420 & $23,00 \mathrm{~S}$ & $50,95 \mathrm{~W}$ \\
\hline Ibirarema & $22,83 \mathrm{~S}$ & $50,06 \mathrm{~W}$ & 471 & $22,75 \mathrm{~S}$ & $50,20 \mathrm{~W}$ \\
\hline Palmital & $22,80 \mathrm{~S}$ & $50,23 \mathrm{~W}$ & 501 & $23,00 \mathrm{~S}$ & $50,20 \mathrm{~W}$ \\
\hline Pedrinhas Paulista & $22,80 \mathrm{~S}$ & $50,78 \mathrm{~W}$ & 330 & $22,75 \mathrm{~S}$ & $50,70 \mathrm{~W}$ \\
\hline
\end{tabular}

Fonte: CIIAGRO/IAC: Centro Integrado de Informação Agrometeorológicas/Instituto Agronômico de Campinas e TRMM.

Por meio de análise de regressão linear avaliou-se a correlação entre os dados estimados pelo satélite TRMM com dados observados nas estações convencionais, e também foram utilizados os indicadores estatísticos: coeficiente de determinação $\left(\mathrm{R}^{2}\right)$, que indica o quanto da variável dependente foi explicado pela variável independente e o índice de concordância "d" (WILLMOTT et al., 1985), para avaliar o ajuste do modelo em relação aos dados observados.
O erro médio absoluto (EMA), expresso na equação 1 , é a medida da magnitude média das diferenças dos valores estimados com os observados.

$$
M A E=\left\lfloor n^{-1} \sum_{i=1}^{n}\left|e_{i}\right|\right]
$$

em que

n =é o numero de resíduos, e $e_{i}=$ número de desvios

\section{REVENG


O erro médio aleatório (não sistemático) (Ea), decorre de fatores imprevisíveis, são flutuações, que fazem com que aproximadamente a metade das medidas realizadas esteja desviada para mais, e a outra metade esteja desviada para menos, afetando a precisão da medida. Precisão é quando pressupõe-se que, se a medida for repetida várias vezes, a variação da mesma em relação ao valor médio medido é baixa.

O erro médio sistemático (Es) que são causados por fontes identificáveis, e em princípio podem ser eliminados ou compensados. Estes erros fazem com que as medidas feitas estejam consistentemente acima ou abaixo do valor real, prejudicando a exatidão da medida. O índice "d" quantifica numericamente a exatidão (coeficiente de concordância), também indica como o modelo simula os valores observados, numa escala de 0 a 1 o grau do desvio da linha 1:1 em uma figura , ou seja, indica o grau de concordância ou exatidão entre os valores estimados e observados, sendo que quando mais próximo de 1 , melhor a exatidão do desempenho do modelo em prever a variável dependente. $\mathrm{O}$ índice é dado pela seguinte expressão:

$$
d=1-\left[\frac{\sum_{i=1}^{N}(P i-O i)^{2}}{\sum_{i=1}^{N}(|P i-O|+|O i-O|)^{2}}\right]
$$

em que

$\mathrm{Pi}=$ é o valor estimado;

$\mathrm{Oi}=\mathrm{o}$ valor observado;

$\mathrm{O}=$ a média dos valores observados.

\section{RESULTADO E DISCUSSÃO}

Os valores apresentados no Quadro 2, indicam de forma quantitativa as relações entre os dados observados e os estimados para os municípios da região estudada. Foram observados valores de "R2", "d" e "EMA", "Ea" e "Es", no período de 1998 à 2008.

Os valores do índice de concordância "d" para a região de Cândido Mota apresentaram resultados precisos entre os dados de precipitação pluvial observados e os dados estimados. Os anos de 1999, 2000, 2001, 2003, 2004, 2005, 2006 apresentaram valores de "d" em torno de 0,80 , sendo que as melhores estimativas ocorreram no ano de 2007 com índice de 0,92, demonstrando ótimo desempenho dos dados analisados. O menor valor de índice "d" foi 0,73 no ano de 2008. Em relação aos erros absolutos médios (EMA), apresentou baixos valores nos anos de 1999, 2000 e 2007, quanto aos Es e Ea, apresentaram baixos valores em todo o período.

Para a região de Campos Novos Paulista, também apresentou bons resultados de índice "d", com valores em torno de 0,80 para os anos de 1999 , 2000, 2001, 2003, 2004, 2005 e 2007, os anos de 1998, 2002 e 2008 apresentaram valores mais baixos, e os erros médios mostraram resultados que podem ser considerados satisfatórios.

A região de Assis apresentou melhor desempenho no ano de 1999, com índice "d" igual a 0,91 , mostrando boa confiabilidade nos dados estimados. Os demais anos apresentaram em média valores de "d" em torno de 0,80 e os erros foram baixos para todo o período analisado. Para o município de Florínea os valores de índice "d" foram acima de 0,80 para todos os anos, com exceção ao ano de 2008, com valor igual a 0,58.

Corroborando com Bardin et al. (2010) e Silva, (2012), que desenvolveram os mesmo estudos para diferentes regiões do estado de São Paulo e obtiveram resultados de "d" em torno de 0,8 e 0,9 , mostrou-se que os dados de precipitação pluvial estimados pelo satélite TRMM podem ser utilizados na ausência de dados pluviais das estações convencionais de superfície.

Os valores de $\mathrm{R}^{2}$ não foram tão elevados quanto os de índice "d", para todo o período analisado, segundo Camargo et al. (2005), isso ocorre devido a precipitação pluvial apresentar uma alta variabilidade espacial. E embora testados os dois tipos de análises (" $\mathrm{R}$ " e "d") a mais indicada nesse estudo foi o "d" de Willmot, devido a escala dos dados serem diferentes, uma pontual (estação meteorológica) e a outra com escala de $0,25^{\circ}$ (satélite TRMM), desta forma os valores obtidos quando comparados dados estimados com os observados, apresentaram valores satisfatórios. 
Quadro 2. Análise estatística dos valores de precipitação pluvial (mm) estimados pelo TRMM e medidos no posto meteorológico

\begin{tabular}{|c|c|c|c|c|c|c|c|c|c|c|c|}
\hline & \multicolumn{11}{|c|}{ Anos } \\
\hline Índices & 1998 & 1999 & 2000 & 2001 & 2002 & 2003 & 2004 & 2005 & 2006 & 2007 & 2008 \\
\hline \multicolumn{12}{|c|}{ Candido Mota } \\
\hline $\mathrm{d}$ & 0,75 & 0,86 & 0,82 & 0,83 & 0,77 & 0,87 & 0,84 & 0,87 & 0,81 & 0,92 & 0,73 \\
\hline $\mathrm{R}^{2}$ & 0,31 & 0,56 & 0,48 & 0,50 & 0,36 & 0,61 & 0,50 & 0,63 & 0,46 & 0,71 & 0,34 \\
\hline EMA & 27,15 & 17,42 & 17,10 & 21,82 & 21,73 & 20,32 & 22,78 & 22,33 & 20,20 & 16,02 & 24,06 \\
\hline $\mathrm{Ea}$ & 35,50 & 24,19 & 24,80 & 25,44 & 27,99 & 27,03 & 31,61 & 25,71 & 28,45 & 23,83 & 33,58 \\
\hline Es & 16,18 & 15,50 & 16,45 & 10,20 & 15,29 & 16,00 & 14,14 & 21,74 & 19,80 & 7,46 & 10,51 \\
\hline \multicolumn{12}{|c|}{ Campos Novos Paulista } \\
\hline $\mathrm{d}$ & 0,57 & 0,88 & 0,86 & 0,80 & 0,78 & 0,82 & 0,82 & 0,89 & 0,76 & 0,80 & 0,42 \\
\hline $\mathrm{R}^{2}$ & 0,13 & 0,61 & 0,57 & 0,45 & 0,43 & 0,49 & 0,44 & 0,70 & 0,35 & 0,44 & 0,00 \\
\hline EMA & 31,71 & 20,97 & 17,28 & 22,18 & 23,67 & 19,91 & 20,02 & 17,76 & 21,54 & 21,34 & 40,01 \\
\hline $\mathrm{Ea}$ & 53,55 & 27,85 & 25,21 & 27,17 & 29,88 & 26,54 & 27,54 & 25,63 & 32,12 & 32,90 & 30,40 \\
\hline Es & 23,62 & 15,25 & 11,81 & 19,12 & 27,36 & 5,84 & 13,55 & 12,63 & 18,33 & 20,99 & 49,21 \\
\hline \multicolumn{12}{|c|}{ Assis } \\
\hline $\mathrm{d}$ & 0,70 & 0,91 & 0,87 & 0,80 & 0,80 & 0,80 & 0,87 & 0,87 & 0,85 & 0,74 & 0,82 \\
\hline $\mathrm{R}^{2}$ & 0,23 & 0,71 & 0,63 & 0,43 & 0,45 & 0,45 & 0,60 & 0,57 & 0,55 & 0,40 & 0,46 \\
\hline EMA & 32,36 & 15,05 & 19,23 & 22,57 & 21,71 & 22,25 & 18,36 & 22,33 & 17,89 & 22,49 & 19,03 \\
\hline $\mathrm{Ea}$ & 40,71 & 22,22 & 22,07 & 26,82 & 29,19 & 27,70 & 22,81 & 29,10 & 22,74 & 34,01 & 25,00 \\
\hline Es & 13,89 & 3,90 & 17,60 & 14,01 & 10,48 & 21,91 & 12,14 & 13,27 & 9,50 & 37,07 & 13,99 \\
\hline \multicolumn{12}{|c|}{ Florínea } \\
\hline$d$ & 0,84 & 0,85 & 0,83 & 0,83 & 0,89 & 0,80 & 0,85 & 0,84 & 0,86 & 0,84 & 0,58 \\
\hline $\mathrm{R}^{2}$ & 0,51 & 0,63 & 0,52 & 0,49 & 0,63 & 0,40 & 0,56 & 0,51 & 0,59 & 0,56 & 0,16 \\
\hline EMA & 23,45 & 19,77 & 20,72 & 20,82 & 15,79 & 20,71 & 19,89 & 24,85 & 17,49 & 18,22 & 24,38 \\
\hline $\mathrm{Ea}$ & 32,49 & 22,41 & 23,87 & 26,49 & 23,06 & 29,54 & 22,16 & 35,53 & 21,01 & 33,36 & 34,12 \\
\hline Es & 6,32 & 23,69 & 21,63 & 15,16 & 5,99 & 10,80 & 14,61 & 7,47 & 12,74 & 9,99 & 18,16 \\
\hline \multicolumn{12}{|c|}{ Ibirarema } \\
\hline $\mathrm{d}$ & 0,78 & 0,89 & 0,84 & 0,82 & 0,79 & 0,83 & 0,87 & 0,82 & 0,77 & 0,79 & 0,87 \\
\hline $\mathrm{R}^{2}$ & 0,36 & 0,65 & 0,53 & 0,48 & 0,46 & 0,50 & 0,57 & 0,47 & 0,41 & 0,46 & 0,57 \\
\hline EMA & 29,20 & 15,17 & 18,73 & 20,12 & 23,98 & 19,78 & 18,78 & 25,97 & 23,17 & 22,37 & 17,90 \\
\hline $\mathrm{Ea}$ & 37,24 & 24,17 & 25,00 & 25,59 & 29,00 & 26,43 & 23,49 & 32,33 & 25,81 & 32,33 & 22,35 \\
\hline Es & 17,84 & 5,36 & 14,39 & 18,50 & 26,99 & 4,78 & 11,11 & 15,95 & 27,14 & 30,46 & 8,32 \\
\hline \multicolumn{12}{|c|}{ Palmital } \\
\hline $\mathrm{d}$ & 0,77 & 0,89 & 0,74 & 0,75 & 0,87 & 0,76 & 0,77 & 0,67 & 0,76 & 0,88 & 0,74 \\
\hline $\mathrm{R}^{2}$ & 0,34 & 0,63 & 0,36 & 0,32 & 0,62 & 0,36 & 0,39 & 0,28 & 0,39 & 0,61 & 0,33 \\
\hline EMA & 24,01 & 15,67 & 23,28 & 25,42 & 19,28 & 25,56 & 25,82 & 31,73 & 23,13 & 21,89 & 20,81 \\
\hline $\mathrm{Ea}$ & 36,67 & 22,64 & 28,08 & 30,00 & 22,45 & 30,85 & 29,93 & 29,68 & 27,29 & 32,52 & 26,97 \\
\hline Es & 11,30 & 7,82 & 25,34 & 13,92 & 17,63 & 21,72 & 24,42 & 38,10 & 27,19 & 9,80 & 9,02 \\
\hline \multicolumn{12}{|c|}{ Pedrinhas Paulista } \\
\hline $\mathrm{d}$ & 0,81 & 0,81 & 0,88 & 0,89 & 0,75 & 0,79 & 0,85 & 0,79 & 0,90 & 0,87 & 0,72 \\
\hline $\mathrm{R}^{2}$ & 0,46 & 0,51 & 0,73 & 0,62 & 0,50 & 0,39 & 0,53 & 0,41 & 0,67 & 0,58 & 0,26 \\
\hline EMA & 28,01 & 23,86 & 19,37 & 16,42 & 24,76 & 24,93 & 18,75 & 26,03 & 15,76 & 20,30 & 22,25 \\
\hline $\mathrm{Ea}$ & 33,30 & 26,80 & 19,18 & 21,92 & 22,78 & 29,09 & 26,16 & 35,10 & 21,44 & 30,73 & 29,55 \\
\hline Es & 20,16 & 28,23 & 22,80 & 8,95 & 37,87 & 19,25 & 11,68 & 15,41 & 7,82 & 12,34 & 11,64 \\
\hline
\end{tabular}


Como mostra o trabalho de Nóbrega (2008), que utilizou os dados estimados do TRMM em uma Sub-Bacia no Amazonas, e os resultados obtidos indicam bons resultados entre os dados de precipitação observados e do TRMM

As Figuras 2, 3, 4, e 5 apresentam a variação decendial dos valores de precipitação pluvial obtidos de estações meteorológicas convencionais, comparadas com os dados estimados pelo satélite TRMM. Nas figuras são mostradas, como exemplo, os municípios de Cândido Mota (Figuras 2 e 3) e Campos Novos Paulista (Figuras 4 e 5), onde se observou que os dados estimados acompanharam as mesmas sequências de valores medidos em estações meteorológicas convencionais.

Em alguns casos observaram-se diferenças consideráveis entre si, como no $5^{\circ}$ decêndio do ano de 2000 e os decêndios 3, 4 e 5 de 2003, no município de Cândido Mota. Diferenças estas também observadas nos decêndios 35 e 36 em 2007 e 2008 para o município de Campos Novos Paulista.

Isso ocorreu devido às diferenças de escalas de cobertura, uma vez que os dados medidos nas estações apresentam escala pontual e os valores estimados pelo TRMM apresentam maior resolução espacial $\left(0,25^{\circ}\right)$, devido a esta diferença de escala notam-se os valores baixos de $\mathrm{R}^{2}$ (Quadro 2).

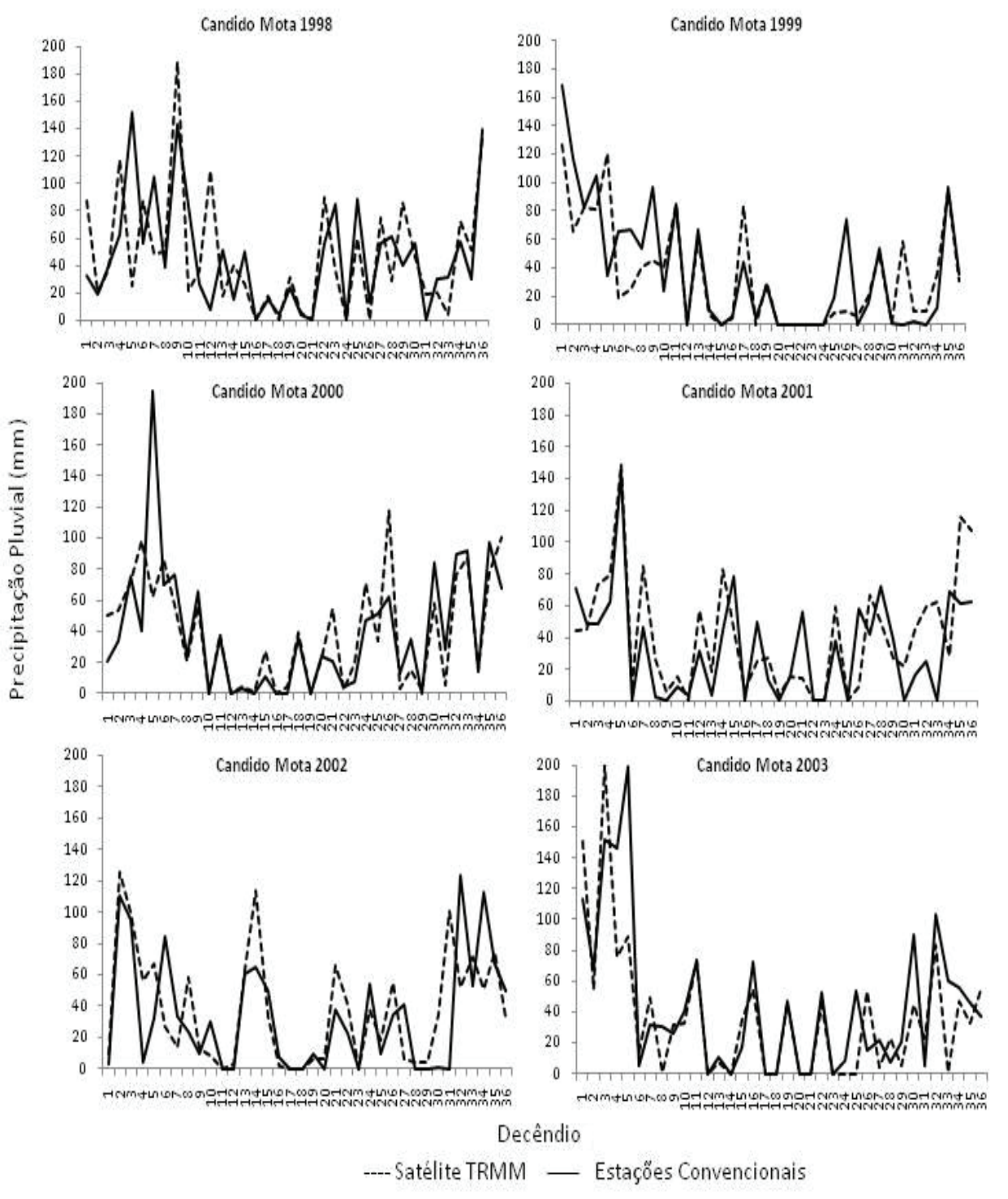

Figura 2. Precipitação Pluvial (mm) observada em estações convencionais e estimada pelo TRMM, período de 1998 a 2003, município de Cândido Mota. 

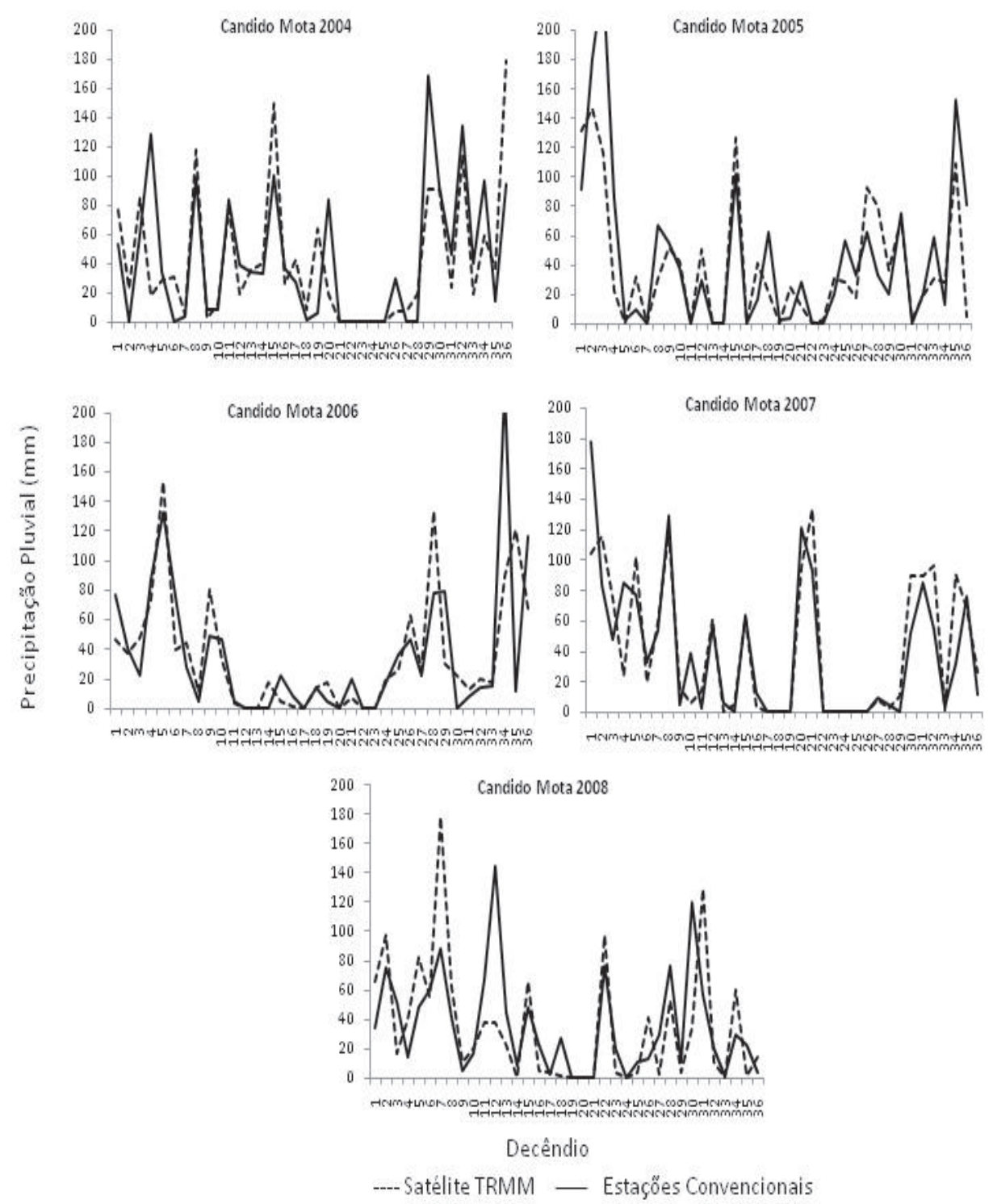

Figura 3. Precipitação Pluvial (mm) observada em estações convencionais e estimada pelo TRMM, período de 2004 a 2008, no município de Cândido Mota. 


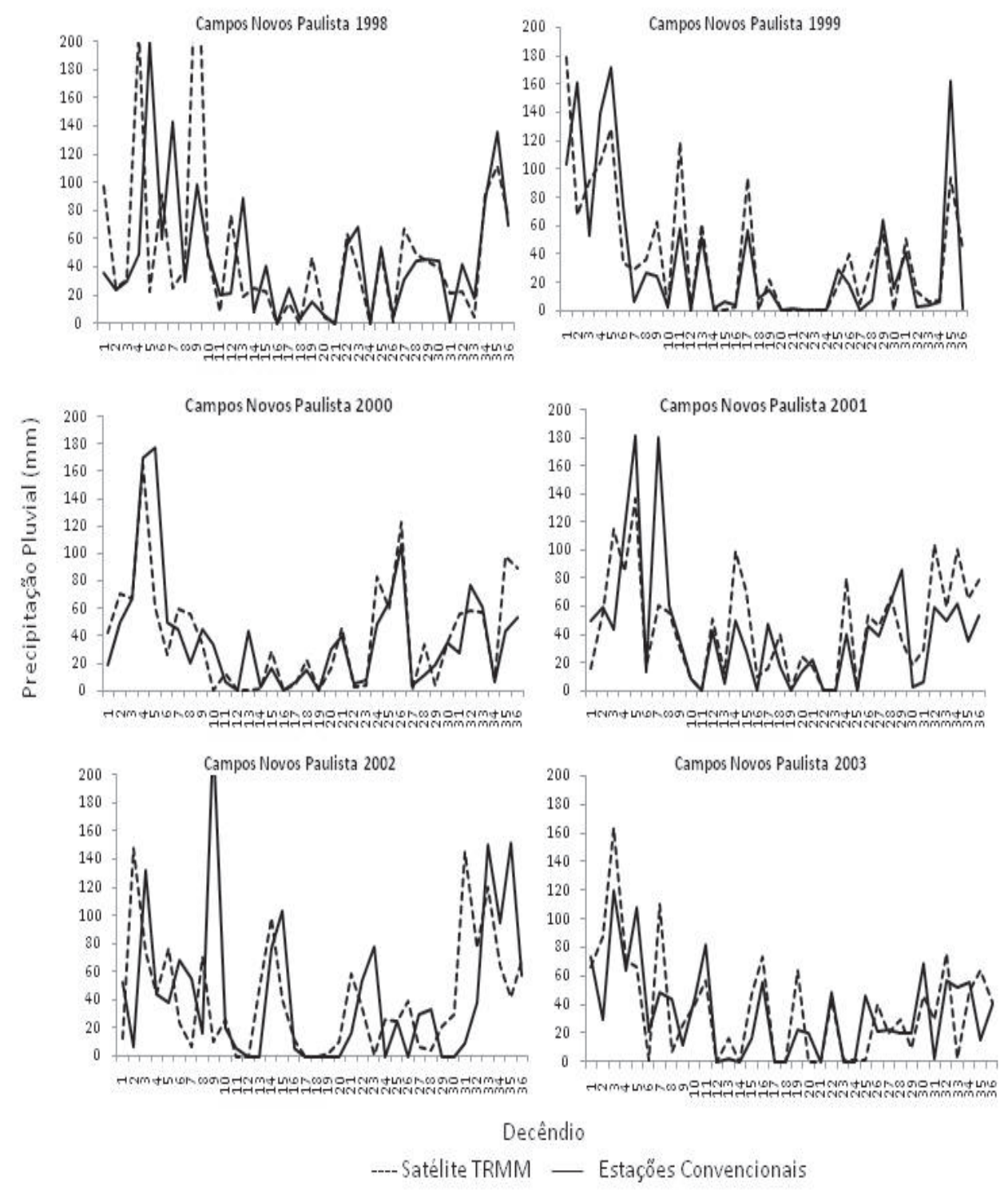

Figura 4. Precipitação Pluvial $(\mathrm{mm})$ observada em estações convencionais e estimada pelo TRMM período de 1998 a 2003, município de Campos Novos Paulista. 

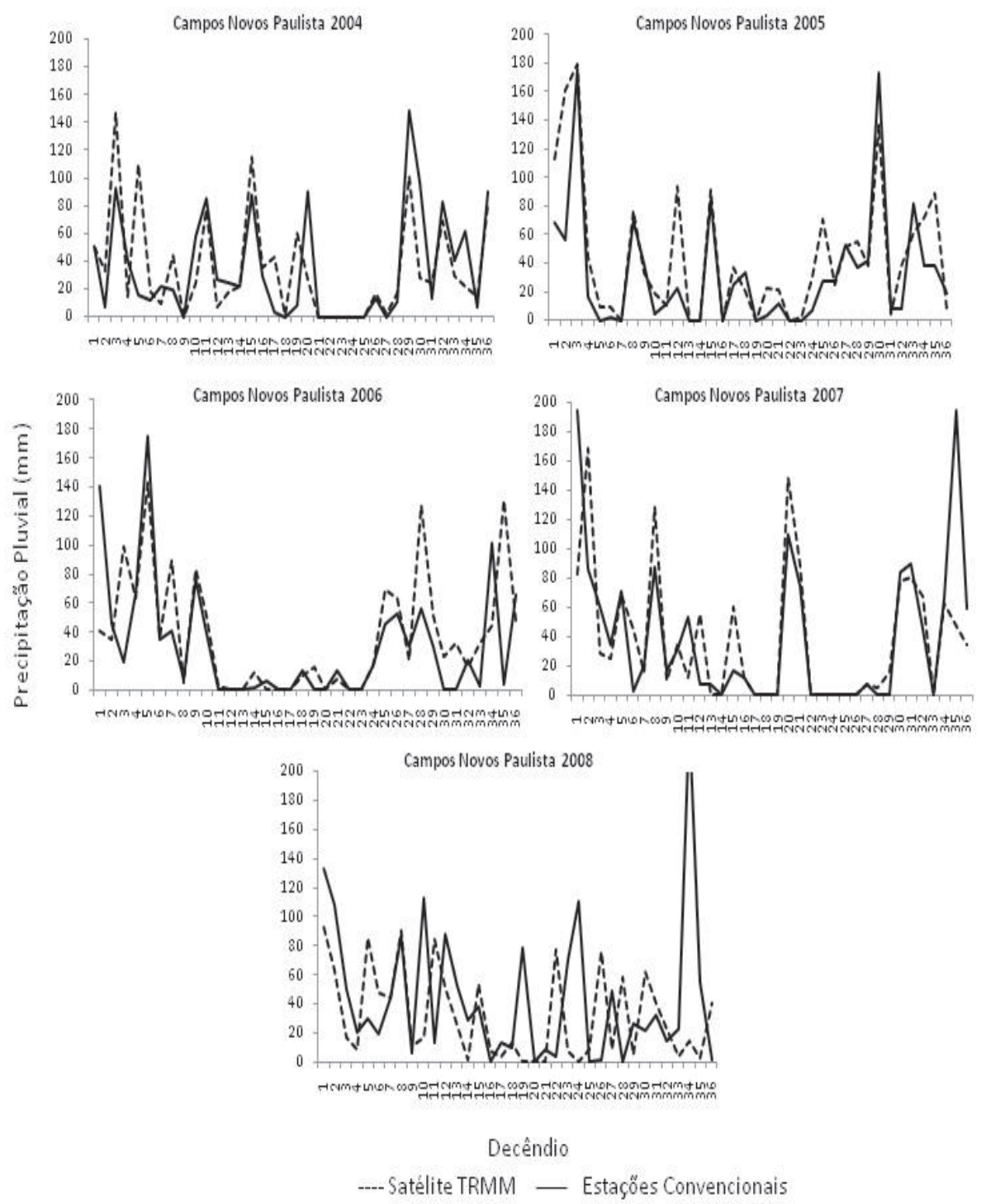

Figura 5. Precipitação Pluvial (mm) observada em estações convencionais e estimada pelo TRMM período de 2004 a 2008, município de Campos Novos Paulista.

\section{CONCLUSÕES}

- O satélite TRMM estimou de forma satisfatória a precipitação pluvial em relação aos dados registrados pelas estações meteorológicas convencionais, apresentando valores de índice "d" de Wilmott acima ou em torno de 0,8 ;

- Os valores de $\mathrm{R}^{2}$ não foram tão elevados, quanto os valores de "d", devido à precipitação pluvial apresentar uma alta variabilidade espacial, e devido a escala dos dados serem diferentes, uma pontual (estação meteorológica) e a outra com escala de $0,25^{\circ}$ (satélite TRMM);

- A utilização de dados de precipitação pluvial estimados pelo satélite TRMM, mostrou que pode ser uma ferramenta, na ausência de informações de superfície, além de servir como entrada de dados para auxílio em modelagem agrometeorológica, monitoramento agrícola, auxílio à pesquisa e para agricultores, por se tratar de dados gratuitos e de fácil acesso.

\section{REFERÊNCIAS BIBLIOGRÁFICAS}

BARDIN, L.; CAMARGO, M.B.P.; BLAIN, G.C.; SILVA,D.F.Comparação entredadosdeprecipitação pluvial observados em estações meteorológicas e estimados pelo satélite TRMM para a região cafeeira da mogiana, São Paulo, Brasil. In: XIII REUNIÓN

\section{REVENG}


ARGENTINA Y VI LATINOAMERICANA DE AGROMETEOROLOGÍA, 2010, Resumos... Bahia Blanca, Argentina: 2010. p.144 -145.

CAGE ,K.S., WILLIAMS, C.R.; CLARK, W.L.; JOHNSTON, P.E.; CARTER, D.A.; Tropical Rainfall Measuring Mission (TRMM) ground validation field campaigns, Journal of Atmospheric and Oceanic Technology, v.19, p.843-863, 2002.

CAMARGO, A.P.; SENTELHAS, P.C. Avaliação do desempenho de diferentes métodos de estimativa da evapotranspiração potencial no Estado de São Paulo, Brasil. Revista Brasileira de Agrometeorologia, Santa Maria, v.5, n.1, p.89-97, 1997.

CAMARGO, M.B.P.; BRUNINI, O.; PEDRO JR, M.J.; BARDIN, L. Variabilidade espacial e temporal de dados termopluviométricos diários da rede de estações agrometeorológicas do Instituto Agronômico (IAC). Bragantia, Campinas, v.64, n.3, p.473-483, 2005.

COLLISCHONN, B.; ALLASIA, D.; COLLISCHONN, W.; TUCCI, C.E.M. Desempenho do satélite TRMM na estimativa de precipitação sobre a bacia do Paraguai superior. Revista Brasileira de Cartografia, Porto Alegre, v.59, n.1, p.93-99, 2007.

BARRERA, D.F. Precipitation estimation with the hydro-estimador technique:its validation against raingage observations., Resumo Anais... In: CONGRESSO DA IAHS, 7.,2005, Foz do Iguaçu.

DUBREUIL, V.; ARVOR, D.; NÉDÉLEC, V.; MAITELLI, G.T. Comparação entre os dados de TRMM, GOES e SPOT-VGT para a estimativa das chuvas em Mato Grosso. In: XIII SIMPÓSIO BRASILEIRO DE SENSORIAMENTE REMOTO, Florianópolis. Resumos... São José dos Campos: INPE, 2007. p.3849-3851.

GARCIA,S.R.; KAYANO, M.T. Início e fim da estação chuvosa na Bacia Amazônica Central: monitoramento com dados de precipitação estimada pelo satélite TRMM. In: III SIMPÓSIO INTERNACIONAL DE CLIMATOLOGIA, 2009, Canela. Resumos... Canela: 2009. p.18-21.

LEIVAS, J.F.; RIBEIRO, G.G.; SOUZA, M.B.; FILHO. J.R. Análise comparativa entre os dados de precipitação estimados via satélite TRMM e dados observados de superfície em Manaus. In: XIV SIMPÓSIOBRASILEIRODESENSORIAMENTO REMOTO, 2009, Natal. Resumos... São José dos Campos: INPE, 2009. p.1611-1616.

LIAO, L.; MENEGUINI, R. Validation of TRMM Precipitation Radar through comparison of its multiyear measurements with ground-based radar. Journal of Applied Meteorology and Climatology, v.48, p.804-817, 2009.

LYU, C.H.; BARNES, W.L. Four years of TRMM/ VIRS on-orbit calibrations and characterization using lunar models and data from Terra/ MODIS. Journal of Atmospheric and Oceanic Technology, v.20, p.333, 2003.

NÓBREGA,S.N. Modelagem de impactos do desmatamento nos recursos hídricos da bacia hidrgráfica do rio Jamari (RO) utilizando dados de superfície e TRMM. 2008 . 186f. Tese (Doutorado em Meteorologia), Universidade Federal de Campinas Grande - UFCG. Campinas Grande, PB.

PRADO, H.; MENK, J.R.F.; TREMOCOLDI, W.A.; JORGE, J.A. Levantamento Pedológico detalhado do Pólo Regional de desenvolvimento tecnológico dos Agronegócios do Médio Paranapanema, Assis (SP). Campinas: Instituto Agrônomico: 2003 (Série Pesquisa APTA. Boletim Científico, 07). p.19. 2003.

SILVA, D.F.da. Uso de Modelos Agrometeorológicos de estimativa de produtividade e risco climático da soja na vale do médio Paranapanema - SP. 2011. 80f. Dissertação (Mestrado em Agicultura Tropical e Subtropical) Instituto Agronômico, Campinas - SP.

SHORT, D.A.; NAKAMURA, K. TRMM radar observations of shallow precipitation over the tropical oceans. American Meteorological Society, Journal Climate, v.13, p.4107-4124. 2000.

TRMM. Tropical Rainfall Measuring Mission. Disponível em: http://trmm.gsfc.nasa.gov/data dir/data.html. Acesso em:_05 set. 2010.

WILLMOTT, C.J.; ACKLESON, S.G.; DAVIS, J.J. Statistics for the evaluation and comparison of models. Journal of Geography Research, v.90, n.5, p.8995-9005, 1985. 